\title{
Les moyens mis en œuvre en Tunisie pour l'étude des crues de la Medjerdah
}

\section{Methods used in carrying out the study of the Medjerdah floods in Tunisia}

\author{
PAR Cl. COLIET
}

INGÉNIEUR, GHEF DE LA SUBDIVISION DES ÉTUdES ET ESSAIS HYDRAULIQUES A TUNIS INGÉNIEUR E.I.H.

\begin{abstract}
Les programmes d'aménagement de l'oucd Medjerdah en Tunisie - lutte contre les inondations, hydroélectricité, irrigation - exigeaient la connaissance approfondie de son régime. Les débits liquides, les débits solides extrêmement importants, l'évolution des fonds, font l'objet de puissantes campagnes de mesure. Présentation de l'appareillage, des méthodes et de l'expérience acquise au cours de ces travaux.
\end{abstract}

Program for the Medjerdah wadi project in Tunisia-struggle against floods, hydroelectricity and irrigation-required increase of knowledge about its characteristics. The discharge, the sediment content (extremely high) and the continual bed changing form the subject of an intensive campaign of measurements. Presentation of the apparatus, methods of use, and experience acquired in practice.

- L'édification de centrales hydro-électriques

le plus important par la dimension de son bassin versant $\left(23.000 \mathrm{~km}^{2}\right)$ et par l'importance de ses apports annuels $\left(900\right.$ millions $\mathrm{de}^{3}$ en moyenne).

Par ailleurs, contrairement à la plupart des autres oueds du pays, son régime connaît une relative régularité.

Ses vallées suffisamment arrosées $\left(^{*}\right)$ connaissent d'ailleurs une prospérité agricole réputée depuis l'époque romaine.

En 1945, un programme d'équipement de la Medjerdah a été entrepris par la Direction des Travaux Publics de Tunisie, destiné à mettre en valeur les ressources encore inutilisées de cette vallée.

Ce programme comprenait en particulier :

- La construction de barrages régulateurs (Oued Mellègue) ou d'alimentation en eau potable (Oued El Lil).

(*) La pluviométrie du bassin versant varie de $320 \mathrm{~mm}$ (Tébessa) à $1.534 \mathrm{~mm}$ (Aïn Draham).
(Nebeur, sur le Mellègue, et Fernana, sur l'Oued El Lil).

- La création de réseaux d'irrigation dans la basse vallée de la Medjerdah.

- L'organisation de la lutte contre les inondations également dans la basse vallée de la Medjerdah.

Des différents points de ce programme, le dernier, en particulier, nécessitait une connaissance approfondie du régime de l'Oued et surtout du comportement de ses crues.

\section{I. - Les problèmes des crues dans la basse vallée de la Medjerdha}

Si la Medjerdah a un étiage très faible $(0,5$ à $1 \mathrm{~m}^{3} / \mathrm{s}$ ) et des eaux moyennes extrêmement paisibles $\left(7 \mathrm{~m}^{3} / \mathrm{s}\right.$ à Djédeida), elle a par contre des crues violentes et rapides qui atteignent à l'en- 
trée de la plaine à Tébourba 1.000 à $1.200 \mathrm{~m}^{3} / \mathrm{s}$ en périodicité décennale.

A Tébourba, la Medjerdah se trouve à la cote 27. Elle a encore $80 \mathrm{~km}$ à parcourir dans une plaine alluviale extrêmement plate, gagnée sur la mer en grande partie. De plus, son lit est embarrassé de nombreux obstacles sous forme de méandres ou sous forme d'ouvrages d'art datant de l'antiquité romaine ou de l'occupation espagnole.

Pour ces raisons topographiques en particulier, à partir de 800 à $900 \mathrm{~m}^{\circ} / \mathrm{s}$, la Medjerdah se met à sortir de son lit en de nombreux points sur ses deux berges à partir de Tébourba.

Au cours de ses débordements, l'Oued a dé- posé sur ses berges une partie des matériaux qu'il charrie et a rehaussé ses rives. Actuellement celles-ci dominent la plaine de 1 à $2 \mathrm{~m}$, do sorte que les eaux d'inondation sont contraintes à stagner dans des «garaats 》 qui se sont formées en plusieurs points.

Par ailleurs, ces inondations rendent frécquemment impraticables la plupart des routes, endommagent les cultures et emportent quelquefois même les animaux ou les hommes.

Parallèlement à des travaux d'assainissement indispensables aux plaines atteintes par les eaux, il était nécessaire d'étudier des rectifications à faire subir au lit pour diminuer les c bordements en fréquence et en intensité.

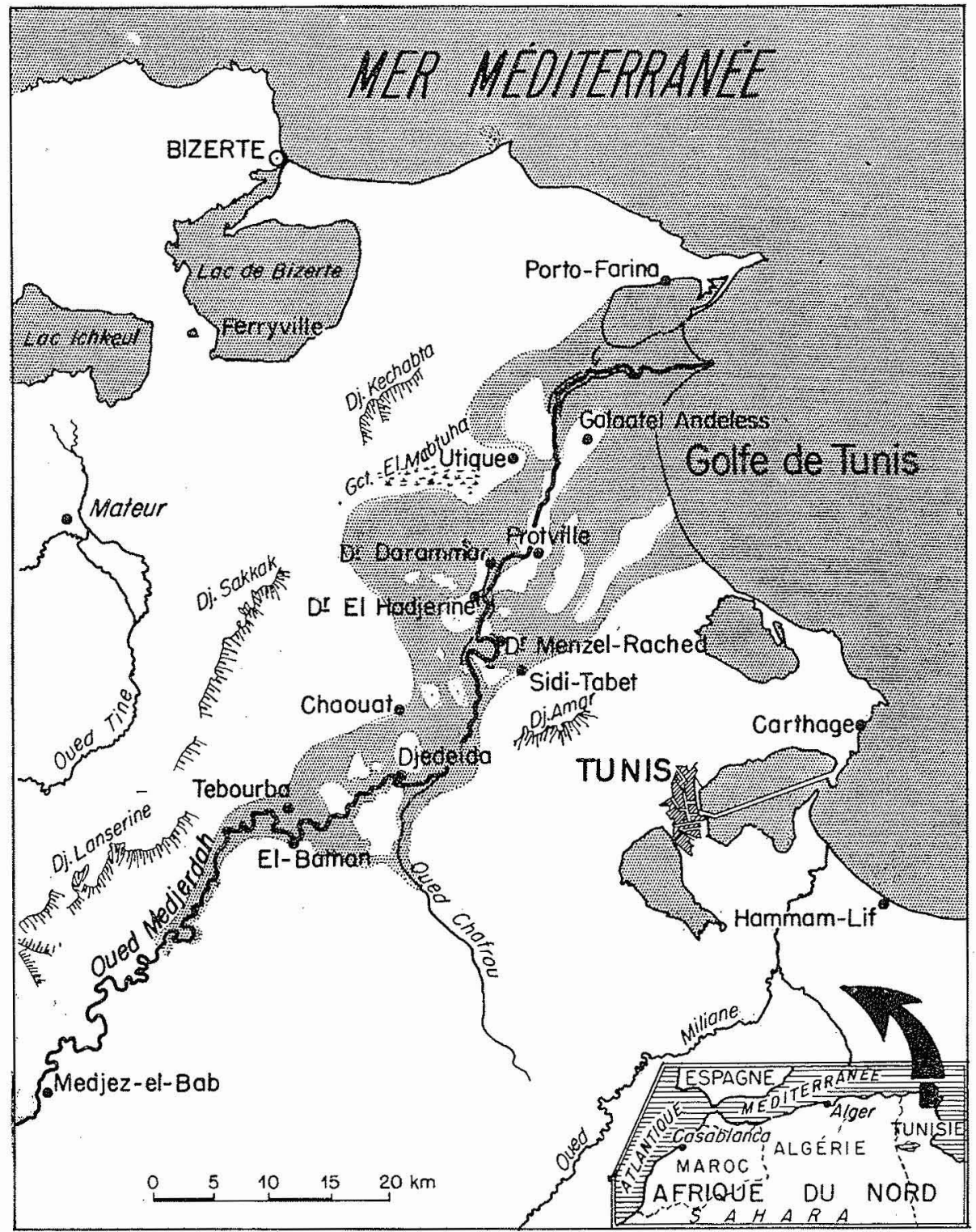

Fig, 1. - Débordements de la Medjerdah. Crue de 1931. 


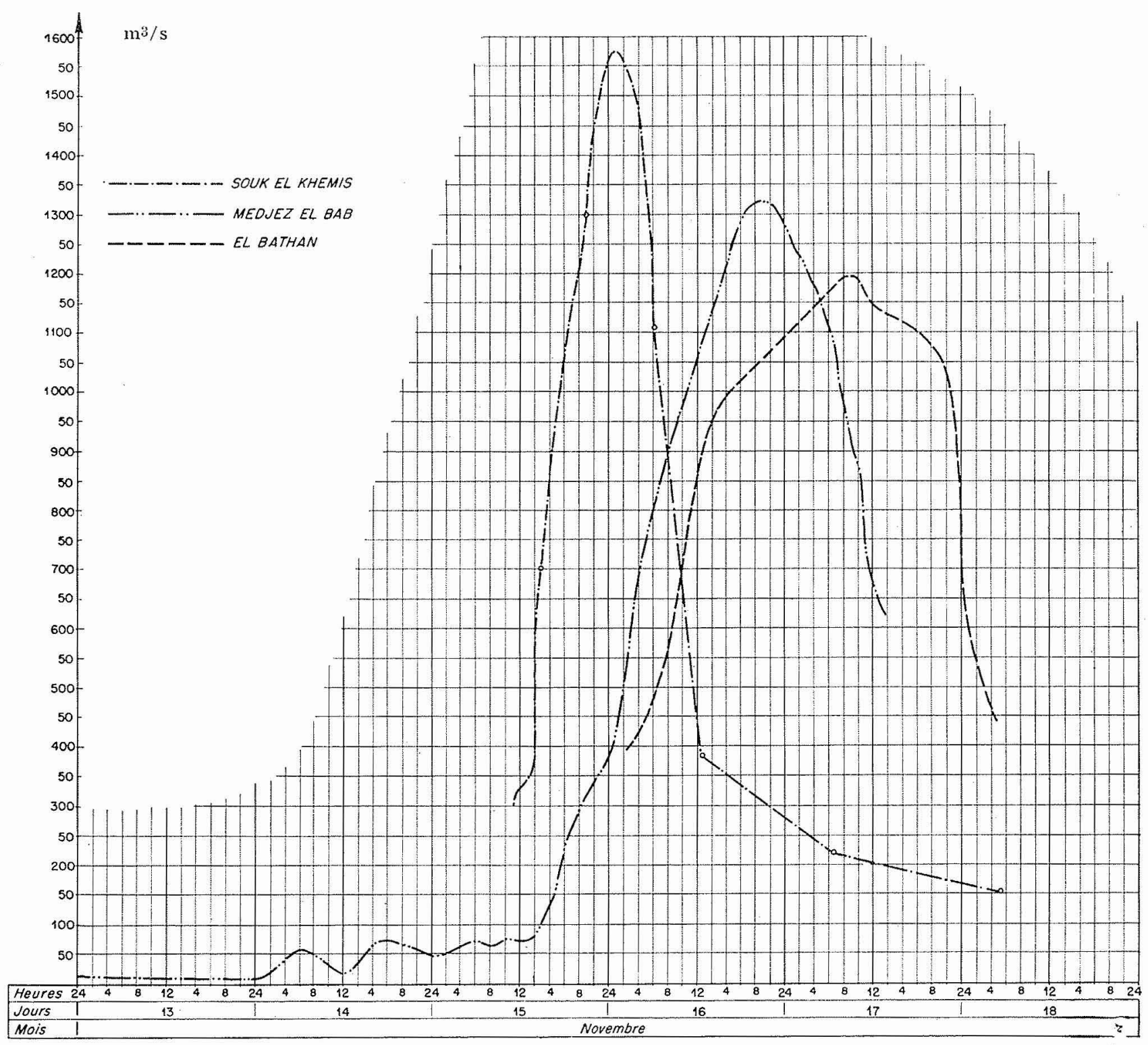

FIG. 2. - Graphiques comparatifs des crues aux divers postes de la Medjerdah en 1936. Crue du 15 au 18 novembre.

Caractéristiques hydrologiques DE LA MEdjerdah

(au poste hydrologique de Medjez El Bab)

Bassin versant...... 20.000 $\mathrm{km}^{2}$

Apport annuel moven.... 915 millions de $\mathrm{m}^{3}$

Débit moyen........... $29 \mathrm{~m}^{3} / \mathrm{s}$

Débit médian ( 1 jour sur 2) $\quad 8 \mathrm{~m}^{3} / \mathrm{s}$

Débit maximum :

de la crue annuelle.... $800 \mathrm{~m}^{3} / \mathrm{s}$ de la crue décennale... $1.200 \mathrm{~m}^{3} / \mathrm{s}$

\section{II. - Les méthodes d'études}

Les principaux moyens de lutte contre les débordements, régularisation du régime, suppression des obstacles (méandres, ouvrages...) ou endiguements, nécessiteront la mise en œuvre de capitaux et de moyens matériels très importants.

Avant d'aborder ces travaux, il était nécessaire d'examiner avec soin quelles seraient les réactions de l'Ơued à ces transformations.

On sait par exemple que, dans certains cas, l'endiguement d'une rivière n'a pour effet que de reporter le problème des débordements à un avenir très proche. La suppression d'une boucle, ou la modification d'un ouvrage, peut entraîner des débordements supplémentaires vers l'aval $\left(^{\star}\right)$. ${ }^{\star}{ }^{\star}$ Ces problèmes sont longuement examinés dans 


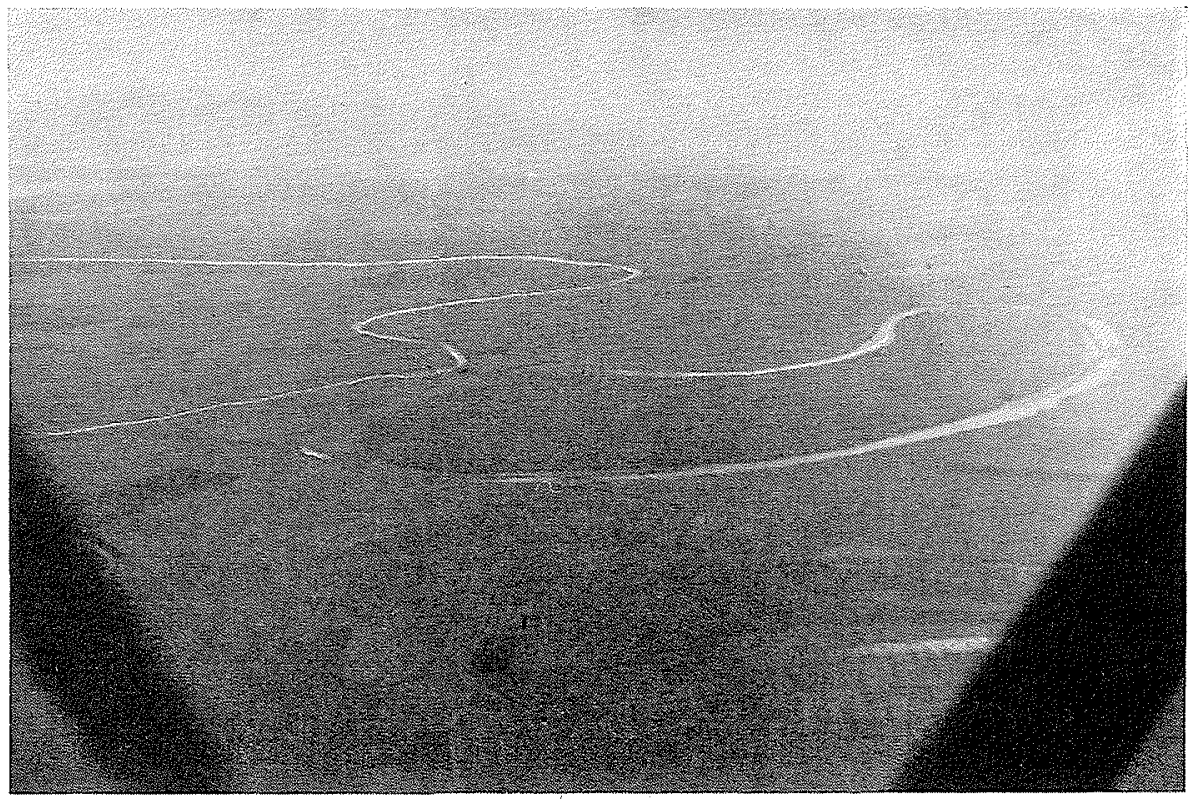

FIG. 3. - Ancien méandro de la Medjerdah en aval de Protville, actuellement abandonné par l'Oued.

L'ensemble du problème du passage des crues, extrêmement complexe, est pratiquement inabordable par le calcul seul. Aussi a-t-il été étudié sur un modèle réduit qui représente la Medjerdah sur les 80 derniers lilomètres de son parcours $\left({ }^{*}\right)$.

$\left(^{\star}\right)$ Ce modèle réduit long de $300 \mathrm{~m}$, un des plus grands d'Europe dans ce genre, a été exécuté et est exploité à Grenoble par le Laboratoire Dauphinois d'Hydraulicque des Etablissements Neyrpic.
Ce modèle, semblable par ses fonctions à une machine à calculer, nous permet, à une échelle de temps réduite, de procéder à des expériences moyennant des frais relativement faibles. Les solutions les plus efficaces et les plus rentables pourront être ainsi essayées avant d'être abordées sur le terrain.

Mais le modèle avait besoin, pour son établissement et sa mise au.point, d'un certain nombre

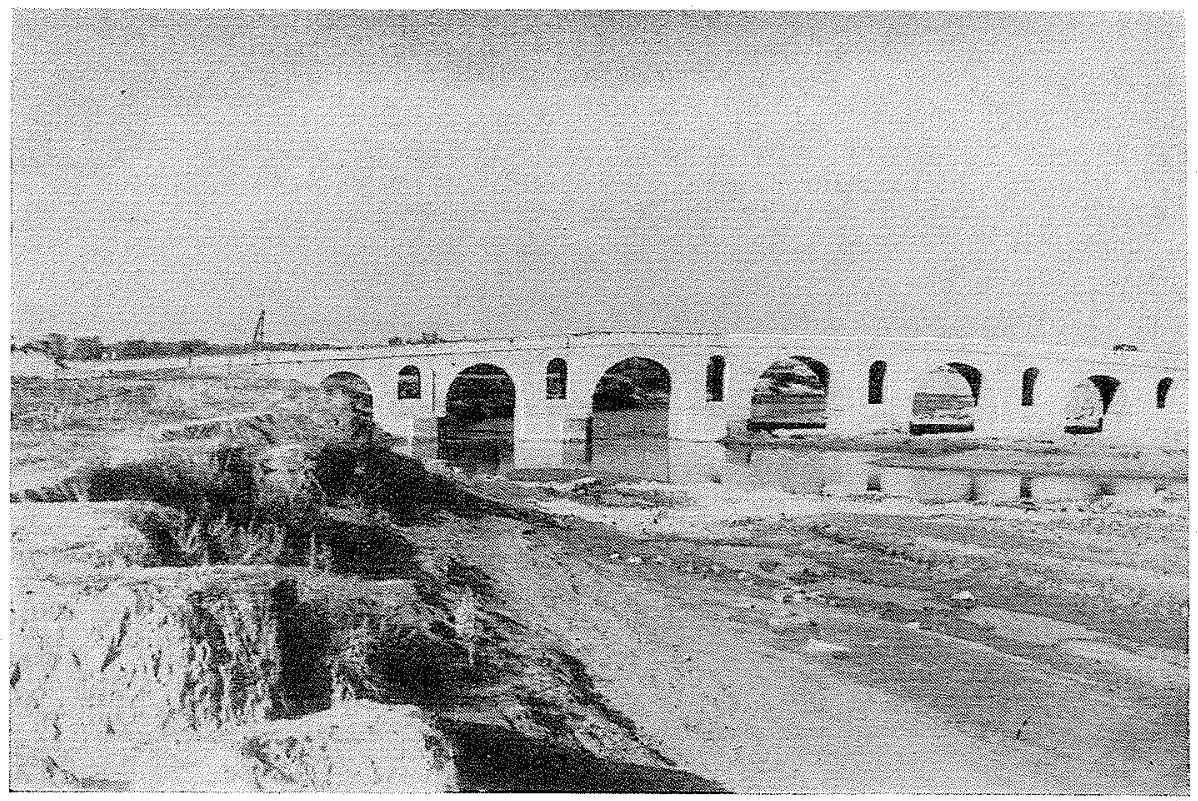

FiG. 4. - Pont antique de Protville. Ce pont at la boucle de Protville créent une obstruction importante. 
de données constructives que l'on peut classer en :

$$
\text { - données topographiques, }
$$

Si les renseignements topographiques sont assez faciles à obtenir, par contre les éléments hydrologiques d'un Oued sont peu aisés à connaître et échappent souvent aux moyens d'investigation simples.

Les principaux problèmes de mesures qui se sont posés au cours de ces études vont être examinés dans ce qui suit.

Nous décrirons, en particulier, les appareils ou méthodes utilisés dans les cas suivants :

$1^{\circ}$ La mesure des débits liquides;

$2^{\circ}$ La mesure des débits solides;

$3^{\circ}$ L'étude des évolutions du lit de l'Oued au cours des crues.

Au moment d'aborder ces études, nous possédions un certain nombre de données statistiques, en particulier en ce qui concerne les débits liquides, qui nous étaient fournies par le Bureau de l'Inventaire des Ressources Hydrauliques de la Direction des Travaux Publics.

Ces données, que l'on devait vérifier par la suite comme très satisfaisantes, ne s'appliquaient malheureusement qu'à des postes (Souk el Khemis et Medjez), situés bien en amont de la zone étudiée, alors que le modèle réduit demandait des étalonnages de la Medjerdah en plusieurs stations situées dans la basse vallée.

Ainsi, la Direction des Travaux Publics fut amenée en 1948 à demander à des spécialistes (*) d'entreprendre ces mesures et d'adapter au cas de la Medjerdah les appareils dont elle disposait.

Actuellement, les premiers résultats obtenus, principalement aux stations de Djédeida, de Protville et d'Henchir 'Tobbias, résutats ayant permis la mise au point du modèle réduit d'études, sont complétés chaque année, au cours des campagnes de mesures, par des équipes des Travaux Publics de Tunisie.

\section{III. - La mesure des débits liquides}

La mesure des débits d'un Oued ne fait pas appel à des techniques nouvelles. Cependant, dans le cas de la Medjerdah, on a été quelquefois amené à utiliser du matériel construit sjécialement pour tenir compte des conditions locales.

La méthode d'évaluation du débit par flotteur de surface peut, dans certains cas, donner de bons renseignements :

On mesure alors uniquement la vitesse superficielle en recherchant l'endroit de l'écoulement où elle est maxima. Le débit est alors calculé par la formule :

$$
\mathrm{Q}=\mathrm{K} \text { V.S. }
$$

S étant la section à l'endroit considéré et $\mathrm{V}$, la vitesse mesurée.

$K$ est un coefficient qui tient compte du fait que la vitesse moyenne dans la section est inférieure à la vitesse en surface.

( ${ }^{\star}$ La Société Neyrpic.

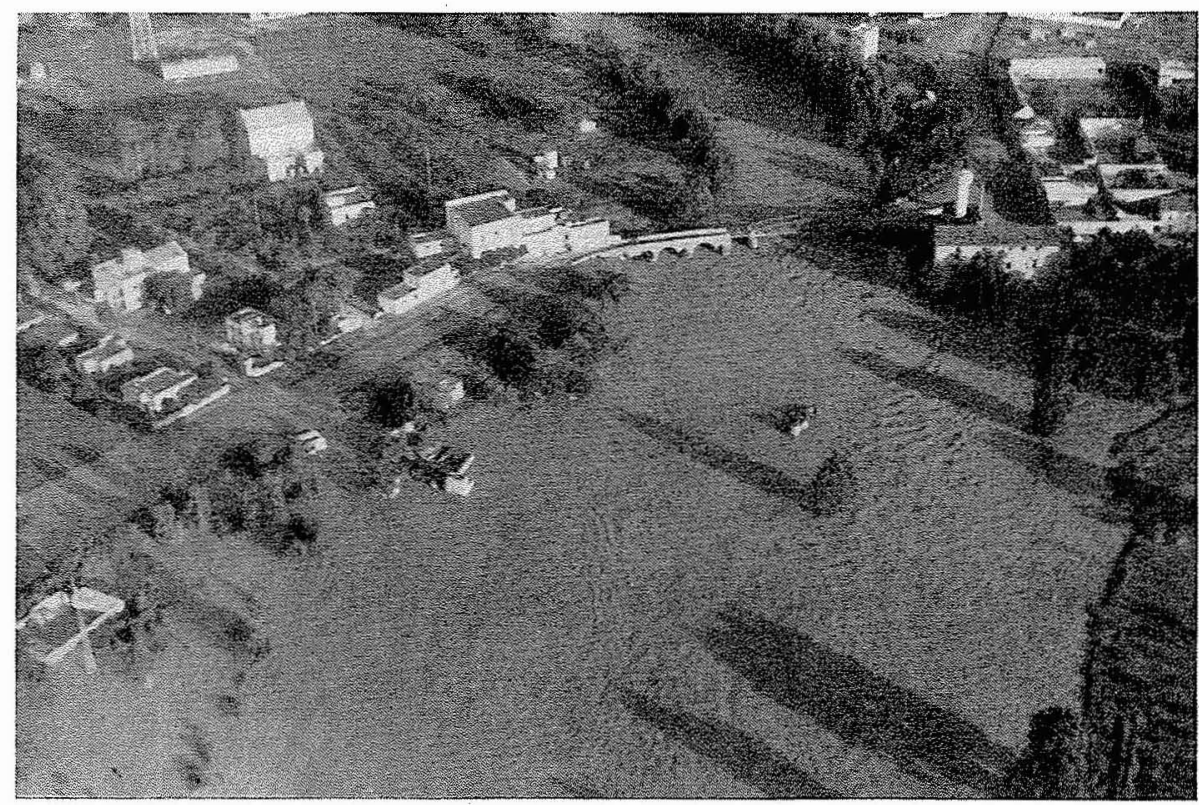

FIG. 5. - Début de débordement à Djédeida. Crue de $850 \mathrm{~m}^{3} / \mathrm{s}$ (novembre 1948). 


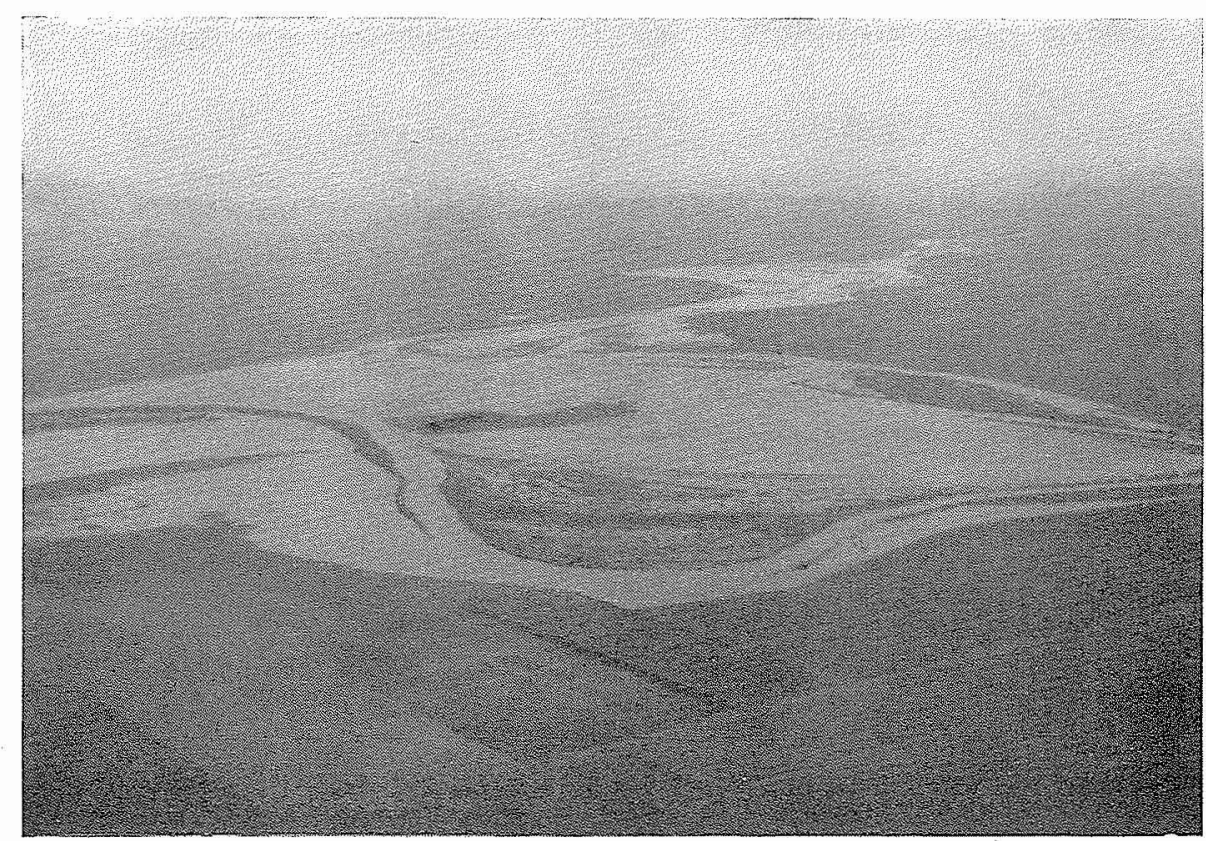

FiG. 6. - Début de débordement dans la boucle de Menzel Rached. (Les eaux sont encore retenues entre les digues.) Crue de $850 \mathrm{~m} 3 / \mathrm{s}$ en novembre 1948.

La valeur de $\mathrm{K}$ varie habituellement entre 0,6 et 0,9 . Certains auteurs donnent des formules pour le calcul de ce coefficient $\left(^{\star}\right)$; mais la va-

(*) Formule de Koechlın :

$$
\mathrm{K}=\mathrm{V}_{m} / \mathrm{V}_{s}=0,82 \frac{(0,6+\sqrt{\mathrm{R})}}{(0,9+\sqrt{\mathrm{R})}}
$$

R étant le rayon hydraulique de la section. leur des résultats reste aléatoire et on ne peut espérer une précision supérieure à 15 ou $20 \%$, d'autant plus que l'erreur sur la détermination de $\mathrm{S}$ peut elle-même être importante.

Cette méthode a cependant l'avantage d'une grande simplicité d'application et elle ne nécessite pas comme les autres systèmes un matériel coûteux et difficile à manier.

Signalons un dispositif original utilisé pour

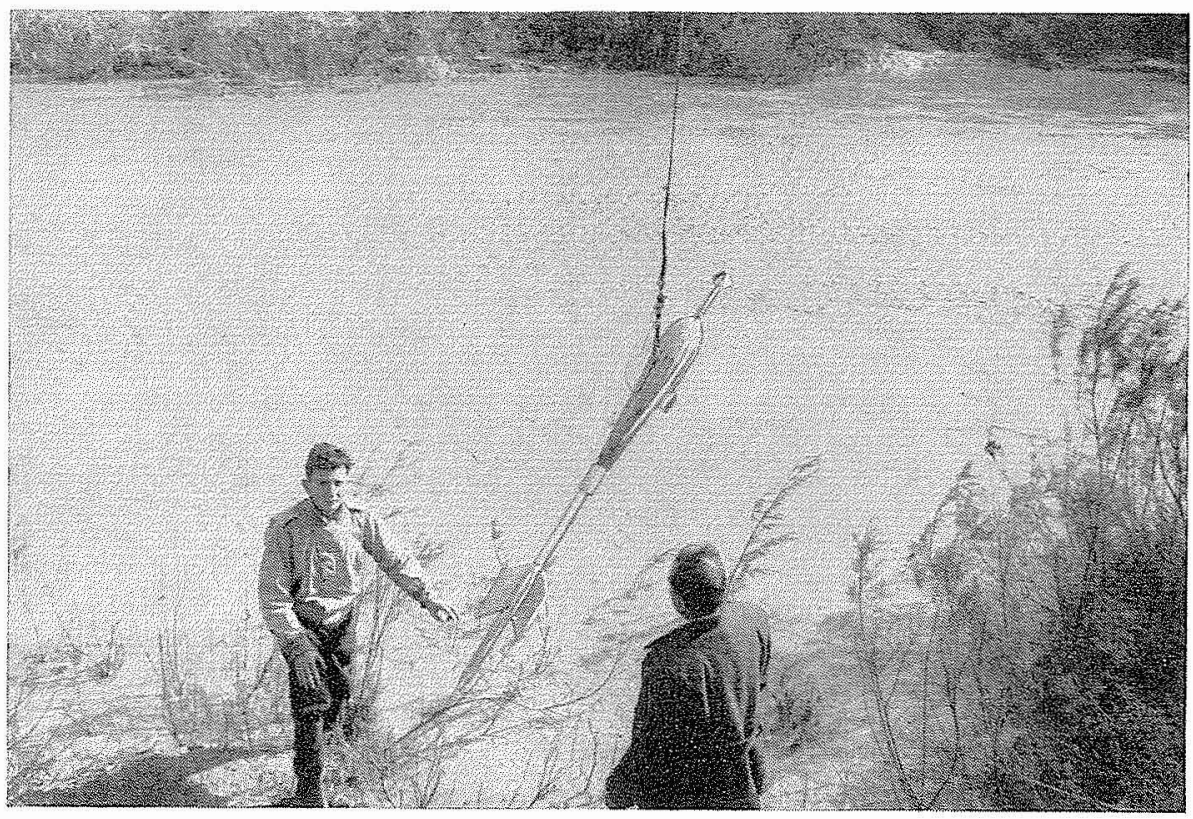

Fic. 7. - «Saumon» porte-moulinet de $80 \mathrm{~kg}$. A l'extrémité du saumon, on aperçoit le moulinet, et à l'opposé la gouverne de direction. 
les opérations de nuit. Il consiste à enduire les llotteurs de peinture « Scotlitt », extrềmement réfléchissante. De cette façon, les flotteurs peuvent ètre suivis à l'aide d'une simple lampe électrique de poche.

\section{LA MESURE DU DÉBIT AU MOULINET}

Le moulinet, fixé à une perche ou placé sur un «saumon» métallique que l'on suspend à un câble, permet de mesurer la vitesse avec une très faible erreur en de nombreux points bien déterminés de la section.

Par intégration graphique, on peut en déduire le débit avec une précision moyenne de l'ordre de $\bar{y}$ à $7 \%$, à condition d'opérer avec soin et de faire un nombre de points de mesures suffisant (1 point pour $10 \mathrm{~m}^{2}$ de section environ, soit de 30 à 40 points dans le cas de la Medjerdah).

Pour l'étude de la Medjerdah, nous avons utilisé surtout des moulinets Dumas.

Cet appareil comprend, comme ses prédécesseurs, une hélice actionnant un contact électrique tous les 10 ou 20 tours.

L'appareil que nous avons utilisé, grâce à une série de dispositifs spéciaux (en particulier des « chambres de décantation ») évite la pénétratioin des limons extrêmement fins de l'eau qui risquent de «gripper » les roulements à billes de l'axe de rotation de l'hélice.

Périodiquement, chaque moulinet est étalonné dans les Laboratoires de la Société Hydrotechnique de France ou de I'E.N.S.E.H. de Toulouse, de facon à surveiller la précision de l'appareil qui est normalement de l'ordre de 1 à $2 \%$.

A cause de l'importance des courants (2 à $5 \mathrm{~m} / \mathrm{s}$ ) et des hauteurs d'eau ( 5 à $10 \mathrm{~m}$ ), il n'a pas été possible, sauf exception, de manipuler les moulinets à partir de perches. On s'est done servi de saumon porte-moulinet et de treuils de manutention.

Des saumons pesant 45 ou $80 \mathrm{~kg}$ ont été nécessaires suivant l'importance de la vitesse pour descendre les moulinets jusqu'au fond des sections.

Lorsque l'on peut, on opère à partir d'un pont : un dispositif à treuils et potence permet une manipulation facile du matériel.

Dans le cas où on a dû opérer en dehors d'un pont, il a alors fallu installer entre les berges une traille téléphérique.

Une traille comprend un câble porteur en acier suspendu à quelques mètres au-dessus des berges. Sur le câble roule un petit chariot. A partir de la berge, à l'aide de deux treuils, on commande les mouvements verticaux et horizontaux du saumon porte-moulinet.

Certains «saumons 》 sont équipés d'un «contact de fond $»$, sorte de palette placée sous le saumon qui établit un contact au moment où l'appareil atteint le fond de l'Oued.

Cependant, on verra plus loin que cette méthode de détermination de la section est, dans le cas qui nous intéresse, insuffisamment précise ou même déficiente, et que l'on doit faire quelquefois appel à des moyens plus perfectionnés.

Pour simplifier les manutentions et surtout

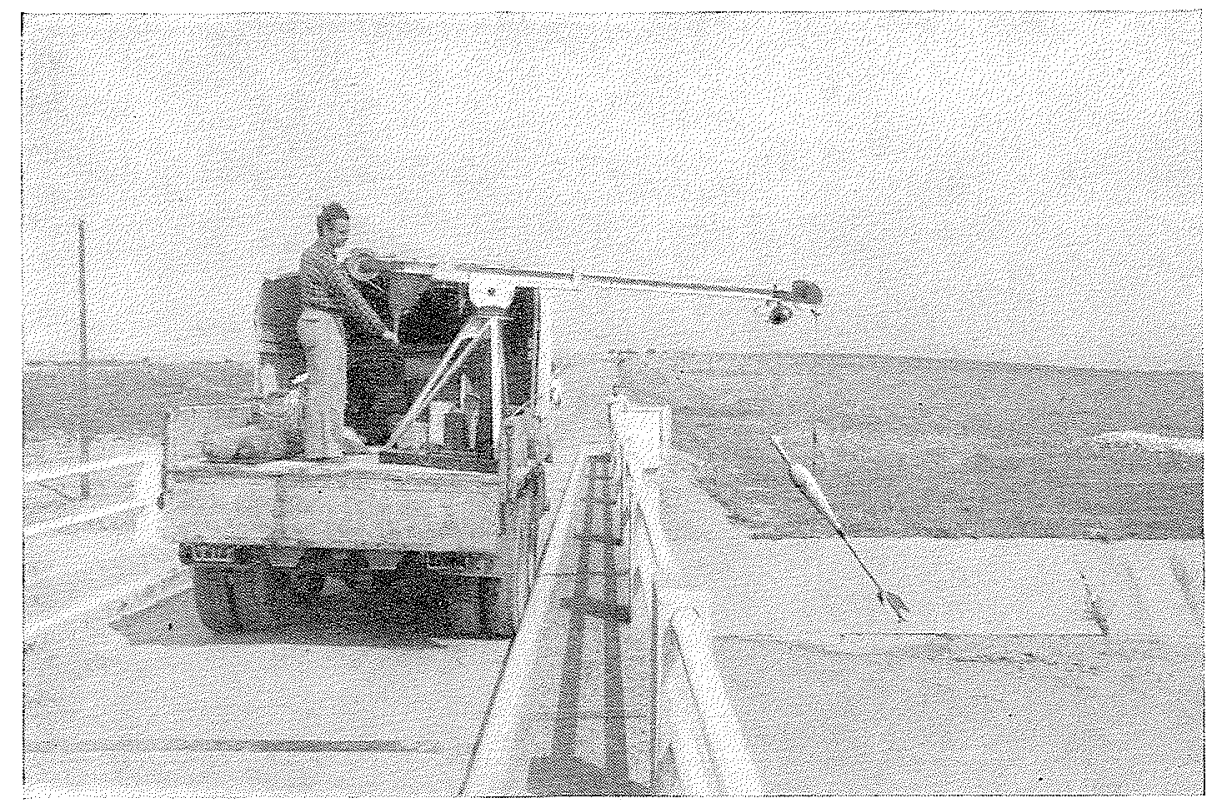

Fra. 8. - Camion équipé pour les opérations de mesures hydrologiques a partir des ponts routes. 
\begin{tabular}{l}
$0 \quad 5 \quad 10 \quad 15$ \\
\hline
\end{tabular}
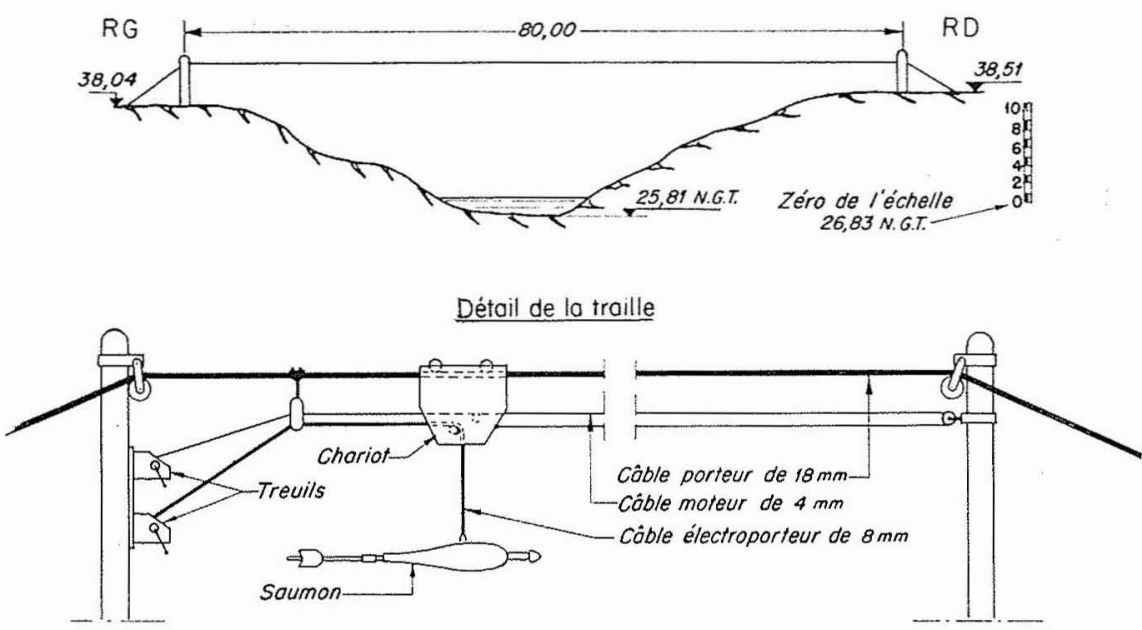

FIG. 9. - Traille de Taullierville.

pour réduire le nombre de câbles immergés dans le courant, on utilise pour la suspension du saumon un câble dit électroporteur, qui contient sous une gaine d'acier un ou deux conducteurs électriques.

En effet, la « traînée » des parties immergées (saumon et câbles) déporte le saumon vers l'aval et donne au câble électroporteur un profil complexe (chainette).

Dans certaines sections (emplacement du bar- rage de Taullierville), l'angle que fait le câble avec la verticale atteint $45^{\circ}\left({ }^{\star}\right)$.

Ainsi la profondeur apparente du saumon, par rapport au plan d'eau, mesurée au compte-mètres du treuil, doit être corrigée en se servant d'abaques pour calculer la profondeur réelle.

(*) L'importance de cet angle était d'ailleurs due en grande partic aux corps flottants venant prendre appui sur le câble.

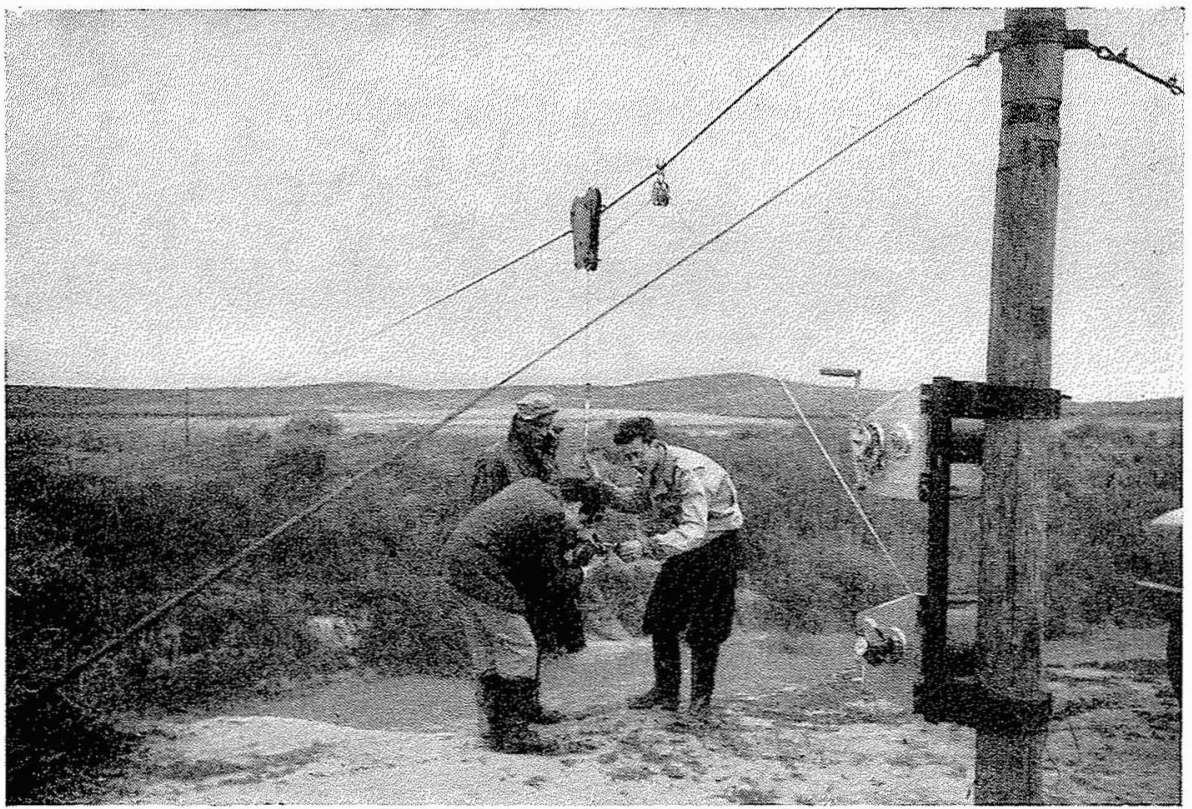

Fig. 10. - Installation de traille téléphérique pour mesures hydrologiques en section libre. On voit sur la photo, au premier plan : les deux treuils de commande; en haut : le chariot de va et vient. 


\section{INTÉGRATION DU DÉBIT}

Pratiquement, que l'on opère d'un pont ou d'une traille téléphérique, on mesure la vitesse du courant le long de verticales régulièrement espacées (5 m en général). Sur chaque verticale, on observe la vitesse à des points espacés de $1 \mathrm{~m}$ environ.

On est ainsi amené, dans une section de 300 à $400 \mathrm{~m}^{2}$, à faire une trentaine de points de mesure, soit un point pour $10 \mathrm{~m}^{2}$ de section environ.

Les résultats des mesures sont portés sur une épure. Par intégration graphique, on détermine successivement :

- La vitesse moyenne sur chaque verticale;

- La courbe des vitesses moyennes de la section;

- La courbe des débits unitaires;

- Par planimétrage de cette dernière, le débit total passant dans la section.

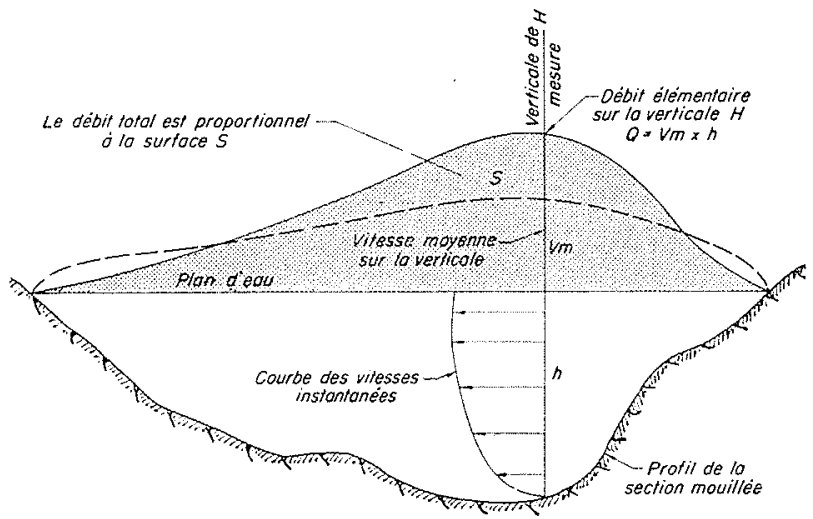

Fıg. 11. - Calcul du débit par intégration graphique.

On a montré que dans de bonnes conditions d'exécution et dans la mesure où l'on respecte les règles énumérées précédemment, la précision peut atteindre $3 \dot{a} 4 \%$. Mais il faudra se méfier avec soin des sections défectueuses ou instables, ou des perturbations dans l'écoulement, ou des évolutions rapides du profil qui peuvent entraîner des erreurs supérieures à $7 \%$.

On conçoit que l'ensemble des opérations de manœuvres et mesures, qui se font fréquemment de nuit et sous la pluie, ne soit pas des plus aisées et nécessite un personnel expérimenté et très entraîné.

\section{LES ÉTALONNAGES ACTUELLEMENT RÉALISES}

Comme nous l'avons dit plus haut, le Burean de l'Inventaire des Ressources Hydrauliques de la Direction des Travaux Publics (B.I.R.H.) a établi depuis plusieurs années un certain nombre de postes de jaugeage.

En particulier, ceux de Souk El Khémis et de Medjez El Bab ont permis d'établir des statistiques sur les débits de la Medjerdah depuis 1932 environ. Ce sont ces résultats que nous faisons paraitre ici sous forme de « courbe des débits classés » de la Medjerdah à Souk El Khémis (lig. 12, 13).

Depuis 1948, nos efrorts ont porté sur l'établissement d'étalonnage de l'Oued en plusieurs points de la basse vallée.

Nous citerons, d'amont en aval :

-- Pont de Borj Toum;

- Emplacement du barrage de Taullierville;

- Pont route de Djédeida;

- La Medjerdah en aval du confluent du Chaffrou;

- Pont route de Protville;

- Pont sur l'émissaire d'Henchir Tobbias;

- La Medjerdah en aval de l'émissaire d'Henchir' Tobbias.

Au total, quatre installations de trailles ont dû être réalisées et dix-huit échelles de lecture de niveau ont été placées sur le bord de l'Oued pour suivre l'évolution des crues.

Chaque année, deux ou trois équipes spécialisées exécutent de 20 à 30 mesures, suivant l'importance des crues.

\section{IV. - La mesure des débits solides}

La mesure des débits solides d'un cours d'eau est sensiblement plus complexe que celle des débits liquides.

D'une part, le problème est en lui-même plus complexe; d'autre part, l'appareillage dont on dispose pour ces mesures est en général compliqué et d'un maniement délicat. Enfin, les analyses que l'on doit faire en Laboratoire sont longues et ne donnent pas tous les renseignements désirés.

\section{LES MÉTHOdES DE MESURE DU DÉBIT SOLIDF:}

Un cours d'eau transporte avec lui une certaine proportion de matériaux solides que l'on peut classer :

$$
\begin{aligned}
& 1^{\circ} \text { en matériaux en suspension, } \\
& 2^{\circ} \text { en matériaux de charriage. }
\end{aligned}
$$

Les matériaux en suspension (les plus fins) sont maintenus dans le courant par la turbulence de l'écoulement. Mais la concentration n'est en général pas uniforme et augmente de 


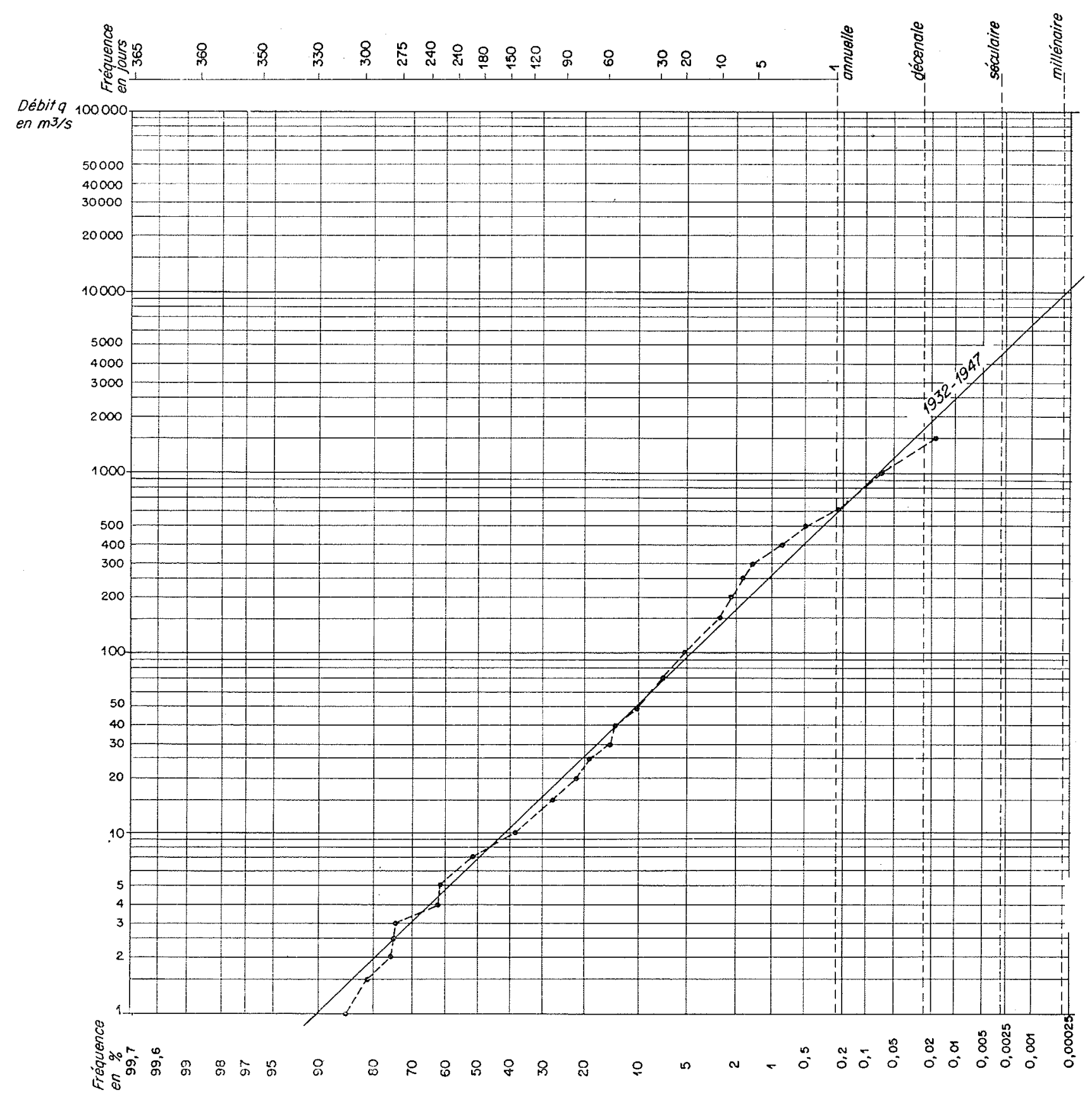

Frc. 12. - Poste de Souk el Khemis. Débit maximum de la journée. Frẻquence. Diagramme Gausso logarithmique.

la surface vers le fond suivant une loi de répartition qui dépend du cours d'eau.

Au voisinage du fond, les matériaux plus lourds qui ne peuvent pas être mis en suspension se déplacent lentement, traînés par les forces de frottement de l'écoulement.

La méthode de mesure la plus simple, qui consiste à prélever un échantillon avec un récipient que l'on immerge dans le courant, ne donnera qu'un ordre de grandeur de la teneur en matières en suspension.
Ces erreurs sont dues en particulier aux formes de l'appareil et aux conditions locales de l'opération.

Un grand nombre d'appareils ont été étudiés et utilisés avec des succès variables sur les rivières européennes.

Quand il s'agit de míesurer le charriage de fond (c'est souvent le cas en Europe), on se sert souvent d'une "nasse », genre de nasse à poisson posée sur le fond du lit et qui ra- 


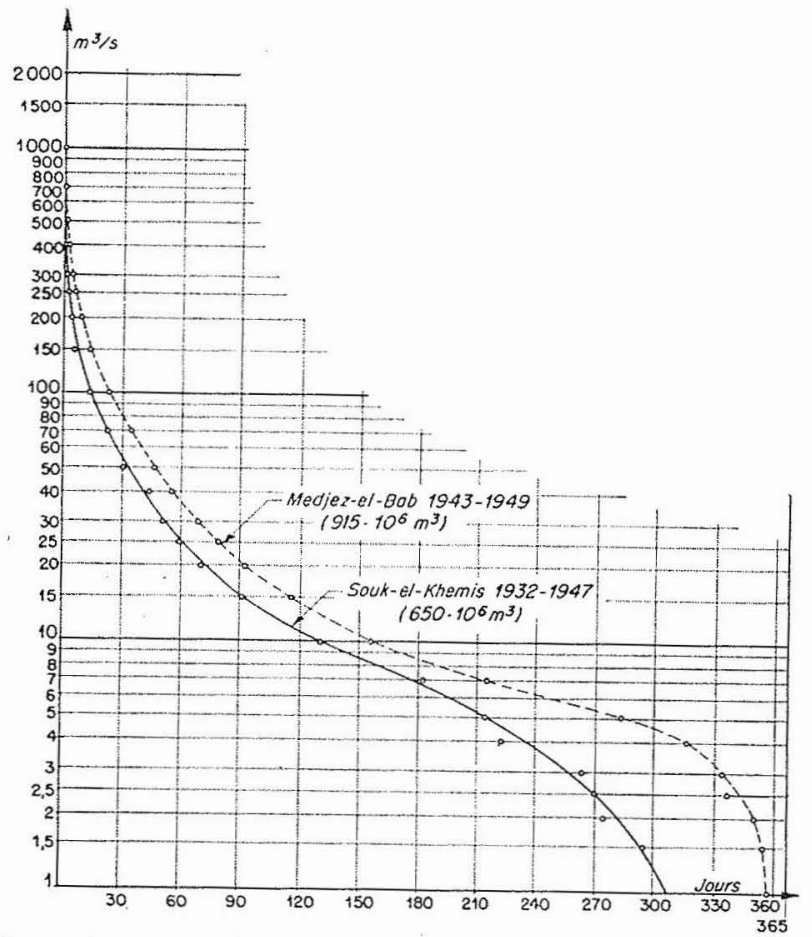

FIG. 13. - Graphique de débits classés d'après le débit moyen de la journée.

masse en un temps connu un certain poids de matériaux.

\section{Prélèvements de matériaux en suspension}

Dans le cas de la Medjerdah, il s'agit surtout de matériaux en suspension.

En effet, les éléments de son lit sont en général très fins et leur dimension ne dépasse guère $0,5 \mathrm{~mm}$.

Il s'agissait donc d'explorer toute la section de l'écoulement pour pouvoir tracer un bilan d'écoulement solide.
Un appareil de prélèvements, pour fournir des prélèvements conformes à la réalité, doit opérer de façon telle que son orifice de prélèvement modifie le moins possible les conditions voisines d'écoulement. En effet, un ralentissement du courant, dû à la présence d'un clapet de fermeture de l'orifice, peut entraîner une modification dans la répartition des matériaux, éliminant par triage les uns ou les autres, donc faussant les concentrations.

La «turbidisonde » que nous avons utilisée, évite, dans certaines limites d'utilisation, ces inconvénients et assure des prélèvements réels. A l'aide d'un treuil, l'appareil est descendu de la même façon qu'un moulinet en plusieurs points de la section. Les différents prélèvements soigneusement étiquetés sont ensuite analysés au Laboratoire de la Direction des Travaux Publics. Celui-ci mesure les teneurs en matériaux en suspension et, dans certains cas, leur granulométrie.

La turbidisonde fonctionne de la facon suivante (fig. 14);

Une bouteille de 1 litre (incluse dans l'appareil) est en liaison avec trois orifices.

$1^{\circ}$ Un ajutage de prélèvement;

$2^{\circ}$ Un orifice d'alimentation en air comprimé;

$3^{\circ}$ Un orifice en relation avec un Venturi place sur le côté de l'appareil.

Le prélèvement s'opère de la façon suivante :

1. Pendant la descente de l'appareil, dans le courant, de l'air comprimé détendu $\left(^{*}\right)$ à partir d'une bouteille d'air à $150 \mathrm{~kg} / \mathrm{cm}^{2}$ du commerce, est injecté dans la turbidi-

(*) A une pression très nettement supéricure à la profondeur d'immersion de l'appareil pour tenir compte de toutes les pertes de charge du circuit (en fait 3 ì $\left.4 \mathrm{~kg} / \mathrm{cm}^{2}\right)$.

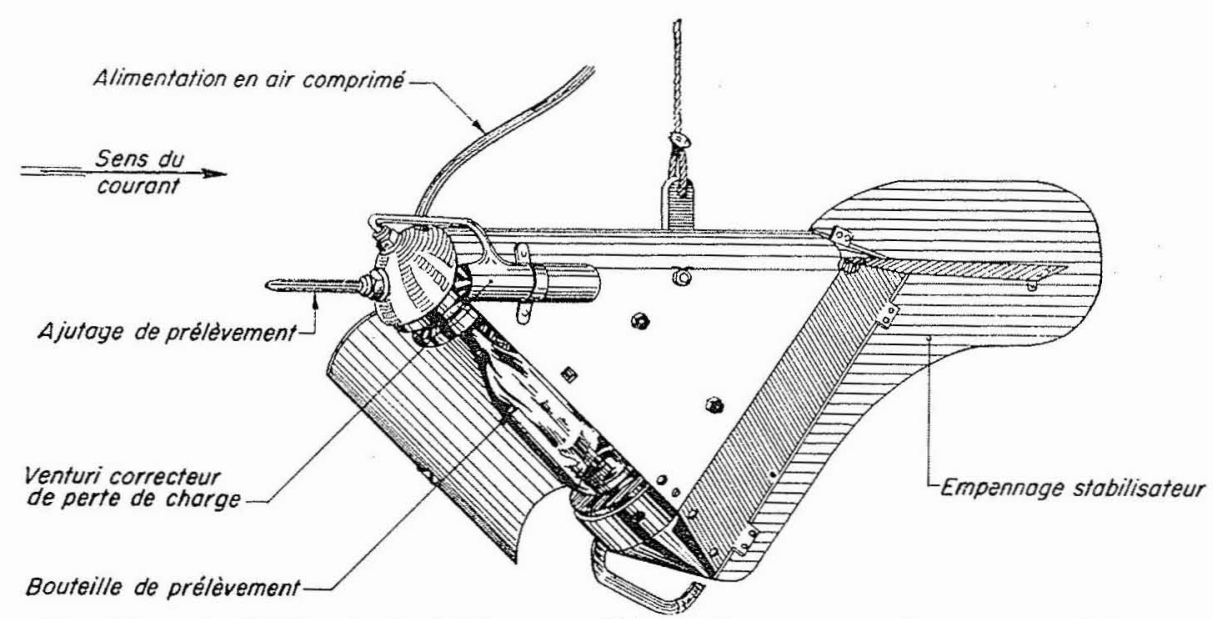

Frg. 14. -- Turbidisonde de 1 litre pour l'étude des concentrations en matériaux en suspension. 
sonde; de cette façon, l'eau ne peut pénétrer dans la bouteille et l'air comprimé débite par les trois orifices.

$2^{\circ}$ La turbidisonde est immobilisée dans l'éconlement. On arrète l'alimentation en air comprimé pendant un temps correspondant au temps de remplissage de la bouteille. Le mélange d'eau et de matières en suspension pénètre par l'ajutage de prélèvement et l'eau s'échappe par le V'cnturi. Ce dernier est calculé pour créer dans la bouteille une légère dépression qui compense les pertes de charge dans l'ajutage de prélèvement.

De cette façon, les vitesses à l'orifice de la prise d'eau sont sensiblement identiques aux vitesses avoisinantes.

3" On arrète le prélèvement en branchant de nouveau l'air comprimé.

Le prélèvement proprement dit dure, suivant la vitesse du courant, de 10 à 30 secondes. Mais l'ensemble de chaque allée et venue durant de 5 à 10 minutes et l'appareil étant relativement lourd, on doit en général se contenter d'une dizaine de prélèvements par section balayée.

\section{Prélèvements de FOND}

Certains modèles de turbidisondes utilisés par nous permettent de s'approcher à quelques centimètres du fond. On a pu constater que la concentration était alors deux à quatre fois plus forte en moyenne que dans le reste de la section. Mais les vitesses d'écoulement étant alors très réduites, le débit solide qui en résulte n'est pas très important.

\section{RÉSUltats ACQUIS}

Le problème des débits solides s'est montré très complexe dans son ensemble.

Nos premières mesures ont mis en évidence:

1. Qu'il existait une répartition à peu près uniforme de la concentration dans la section;

$2^{\circ}$ Qu'en général la concentration augmentait en même temps que le débit;

$3^{\circ}$ Que, pour un même débit liquide, la concentration variait beaucoup d'une crue à l'autre.

Ce n'est évidemment qu'une étude minutieuse, sur un grand nombre de cas, qui permettra vraisemblablement d'établir des relations entre les différents facteurs agissant sur le débit solide

On peut cependant dejà̀ se faire une idée ap- proximative sur ce que la Medjerdah charrie chaque année.

En effet, sur les 915 millions de $\mathrm{m}^{3}$ d'eau que charrie annuellement la Medjerdah, on peut considérer qu'une moitié, correspondant aux eaux des crues, est chargée en moyenne de $25 \mathrm{~g}$ au litre, et que l'autre moitié correspondant aux moyennes et basses eaux, est chargée à $10 \mathrm{~g}$ au litre, soit un total de 16 millions de tomnes de sédiments.

Ceci représenterait approximativement une couche de près de un centimètre d'érosion sur une surface de l'ordre de $1.000 \mathrm{~km}^{2}$.

Par ces chiffres, on se rend compte de l'importance que peuvent prendre ces problèmes de transport des matériaux. Ces sédiments se retrouvent en effet dans le basses vallées, les estuaires et entraînent des évolutions continuelles du lit mineur.

\section{V}

\section{L'étude de l'évolution des fonds du lit}

Comme nous l'avons signalé plus haul, il est difficile au cours des crues de déterminer par sondage au saumon les cotes exactes du fond du lit.

On sait, cependant, que celles-ei évoluent, il est d'ailleurs facile de le constater d'un étiage à l'autre.

Mais pendant la montée de la crue, et au cours de la décrue, la section peut évoluer sensiblement.

D'une part, il en résulte une erreur sur le calcul du débit mesuré dans la section; d'autre part, le comportement des fonds peut être un des facteurs importants agissant sur la ligne d'eau de l'Oued et donc sur les débordements en période de crue.

Lorsque l'on sonde la section à l'aide d'un saumon suspendu à un câble, l'ensemble saumon-câble placé dans le courant est soumis à une force de « traînée ».

Certaines formules tenant compte de la forme que prend̀ le câble permettent de corriger la profondeur apparente indiquée au compte-mètres des treuils. Mais ces formules ne tiennent pas compte évidemment des corps étrangers, tels que branchages, qui viennent's'agglomérer sur les câbles et fausser les calculs dans des proportions indéterminées. Cest donc à un appareil plus perfectionné que l'on a dû faire appel : le sondeur à ultrason. 


\section{LE SONDEUR A ULTRASON}

Le principe des sondeurs à ultrason est maintenant couramment applıqué, principalement pour l'étude des fonds marins, après avoir servi pendant la dernière guerre a la recherche des sous-marins en plongee.

L'appareil comprend, à l'interieur d'un flotteur posé a la surface de l'eau, un émetteur d'ultrason. L'onde se propage dans l'eau à une vitesse très voisıne de $1.000 \mathrm{~m} / \mathrm{s}$ et a la propriété de se réfléchir sur les obstacles rencontrés, comme le font les ondes sonores dans l'air. Une deuxième pièce, également placée dans le flotteur, constitue le récepteur.

L'émission se fait de façon discontinue. Les « signaux»d'émission et de réception, lus sur un oscillographe cathodique, sont séparés par un espace proportionnel au temps du parcours de l'onde sonore et done à la profondeur.

Pratiquement, le cadran de l'oscillographe, parcouru par un balayage circulaire, est graduć en mètres de hauteur d'eau (*).

Dans le dispositif utilisé sur la Medjerdah, l'émetteur-récepteur des signaux ultra-sonores est constitué par un bloc magnéto-strictif au ferro-nickel.

Le «top » d'émission est obtenu par la décharge d'un condensateur dans un circuit oseillant.

Le réglage de cet appareil a surtout consisté à la recherche d'une fréquence convenable pour le cas des eaux de la Medjerdah.

Nous avons vu que ces eaux sont très chargées en particules en suspension susceptibles de créer des échos parasites et done de troubler les résultats.

Finalement, les expérimentateurs ont adopté une fréquence de 35 kilocycles.

Des contrôles de l'appareillage en eau trouble ont montré que l'on pouvait attendre des mesures une précision de l'ordre de $10 \mathrm{~cm}$.

(*) Dispositif adopté sur l'appareil des Etablissements Neyrpic.

\section{LES OPÉRATIONS DE SONDAGES ULTRASON}

Pour relever une section de l'Oued, on peut déplacer le sondeur à partir d'une passerelle ou d'un pont. Mais, en général, on recherche de préférence une section « libre », c'est-à-dire une section qui ne soit pas embarrassée par les piles et culées d'un pont susceptibles de modifier localement les conditions normales d'écoulement.

Nos premières mesures de ce genre, qui datent de 1949, ont été effectuées à partir d'une traille, un peu en aval du confluent du Chaffrou.

Le choix de cet emplacement nous avait été dicté par le modèle réduit de la Medjerdah qui signalait à cette section d'importantes évolutions du fond de l'Oued.

Ce n'est qu'après des observations, au cours de plusieurs crues d'importances diverses, que nous avons pu connaitre avec plus de précision ce point du problème. On a ainsi pu constater

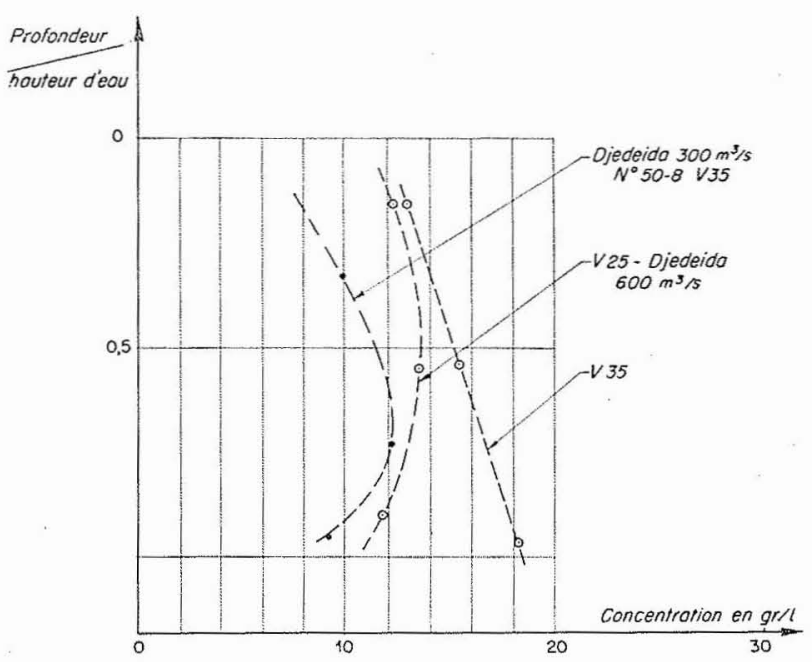

Fig. 15. - Etude de la variation de la concentration en matériaux en suspension en fonction de la hauteur (Poste de Djedeida).

des variations du fond de l'ordre du mètre. Ón comprend dès lors l'importance d'un tel phénomène s'il se généralise sur une partie du cours d'eau.

\section{LA LUTTE CONTRE LES INONDATIONS LES AMÉNAGEMENTS DE LA BASSE MEDJERDHA}

Les premiers esssais sur modèle réduit ont montré l'influence importante des boucles de Menzel Rached et re Protville dans le système des inondations.
En effet, il a été montré qú la suppression de ces deux principaux obstacles permettrait de porter le débit eritique de débordement dans la basse Medjerdah de $800 \mathrm{~m} / \mathrm{s}$ à $1.200 \mathrm{~m} " / \mathrm{s}$, 
c'est-à-dire de réduire la fréquence des inondations dans la proportion de 1 à 5 .

D'autres aménagements, tels que la construction de certaines digues, pourraient vraisemblablement porter le débit critique à $1.500 \mathrm{~m}^{3} / \mathrm{s}$.

Mais chaque modification du cours entraine pendant quelques années des évolutions auxquelles il faut prendre garde.

Par exemple une coupure de boucle, c'est-àdire le raccourcissement du cours, si elle entraîne une amélioration de l'écoulement amont, par abaissement de la ligne d'eau, risque par contre d'entraîner à l'aval une aggravation de la situation.

Par ailleurs, l'ćrosion régressive du lit, conséquence de l'abaissement de la ligne d'eau, peut former plus à l'aval, momentanément, un bouchon défavorable au passage des crues.

Ce n'est done qu'avec beaucoup de pricautions qu'il conviendra de procéder à des modifications du cours d'eau. Dans chaque cas, le modèle apporte des enseignements précieux nécessaires à une bonne coordination des opérations.

Le programme des aménagements de la basse Medjerdah, que l'on entreprendra par l'aval, comprendra principalement les travaux suivants :

1 " Suppression de la boucle et du Pont de Protville;
20 Suppression de la boucle de Menzel Rached;

$3^{\circ}$ Aménagement des anciens ouvrages de Djédeida et d'El Bathan dont les débouchés sont insuffisants;

$4^{\circ}$ Construction de digues aux points bas des berges.

Ces travaux, très importants pour la plupart, devront être échelonnés sur plusieurs années, d'une part pour tenir compte des possibilités de financement, d'autre part pour attendre le plein effet d'une modification avant d'en mettre une nouvelle en service.

Le premier des travaux prérus, la suppression de la boucle de Protville, a débuté en 1952 par la construction d'un nouveau pont de $130 \mathrm{~m}$ de long à faible coefficient d'obstruction. Ce pont est placé sur le tracé du cours rectifié.

La coupure de boucle ne pourra ètre amorcée qu'une fois le pont achevé. Cette amorce sera constituée par un canal pilote de faible section, et ce sont les eaux elles-mêmes de la Medjerdah qui se chargeront d'éroder et d'évacuer les terres du lit jusqu'à création d'une section normale; cette technique très économique a déjà été utilisée pour une rectification du cours de la Medjerdah plus en amont, à Testour, et a donné de bons résultats.

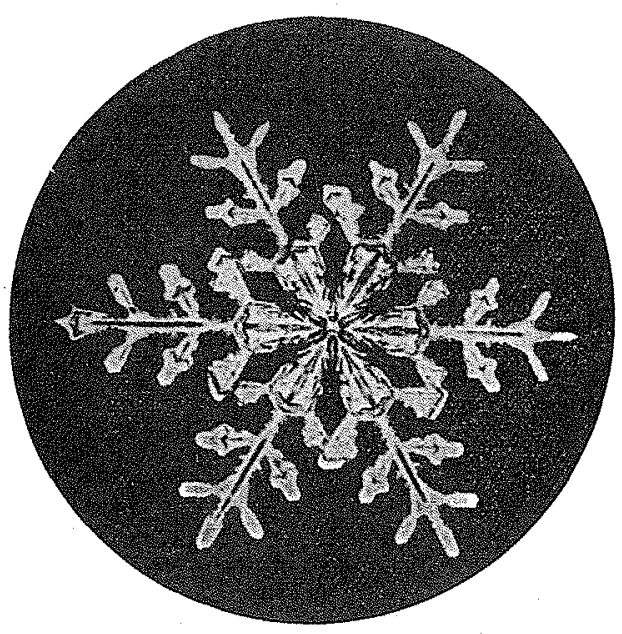

